\title{
Cubic Spline Approximation for Weakly Singular Integral Models
}

\author{
Franca Caliò, Elena Marchetti \\ Dipartimento di Matematica, Politecnico di Milano, Milano, Italy \\ Email: franca.calio@polimi.it, elena.marchetti@polimi.it
}

Received April 10, 2013; revised May 10, 2013; accepted May 17, 2013

Copyright (C) 2013 Franca Caliò, Elena Marchetti. This is an open access article distributed under the Creative Commons Attribution License, which permits unrestricted use, distribution, and reproduction in any medium, provided the original work is properly cited.

\begin{abstract}
In this paper we propose a numerical collocation method to approximate the solution of linear integral mixed VolterraFredholm equations of the second kind, with particular weakly singular kernels. The collocation method is based on the class of quasi-interpolatory splines on locally uniform mesh. These approximating functions are particularly suitable to tackle on problems with weakly regular solutions. We analyse the convergence problems and we present some numerical results and comparisons to confirm the efficiency of the numerical model.
\end{abstract}

Keywords: Volterra-Fredholm Integral Equations; Collocation Methods; Splines

\section{Introduction}

Splines have been used in numerical integration, with all their well known properties, ever since they entered in the numerical analysis scene [1].

In the nineties, splines have been used in more general aspects in numerical integration such as product integration and numerical approximation of models with Cauchy principal value integrals $[2,3]$.

However these results are not completely satisfactory as they use functional values at equally-spaced nodes, whereas in applications it is desirable to densify points in places where the integrand function is not smooth and use fewer nodes where it is. To tackle on this problem, Rabinowitz [4] proposed, with respect to numerical integration, the use of an important class of splines, known as variation diminishing splines (VDS), introduced and investigated, as a tool of approximation theory, in the seventies by Schoenberg [5].

Subsequently, to improve the quality of the approximation, the quasi interpolatory (q.i.) splines, proposed and analysed by Lyche and Schumaker, [6], in different kind of integrals are used, algorithms are given and convergence results are proved in $[7,8]$.

From the second half of the nineties, taking advantage of all these results, the use of q.i. splines in different kind of integral equations is suggested and analysed in [9-12].

In this work we apply a numerical model based on cubic q.i. splines approximation to special mixed Volterra-
Fredholm integral equations of second kind with particular convolution kernels.

In Section 2 we present the mathematical model, in Section 3 we recall the background on q.i. spline space, in Section 4 the numerical method is described, Section 5 is devoted to convergence analysis, finally in Section 6 we show numerical results to complete the theoretical statements and to emphasize the efficiency of the method in the case of solution with discontinuity from the first derivative.

\section{Volterra-Fredholm Integral Equations}

In this paper we consider the following Volterra-Fredholm integral equation:

$$
\lambda u(x)=f(x)+\int_{0}^{x} k_{1}(x, s) u(s) \mathrm{d} s+\int_{0}^{1} k_{2}(x, s) u(s) \mathrm{d} s(1)
$$

where $u:[0,1] \rightarrow \mathbb{R}$ is the unknown function, $f(x)$ is a known function such that $f \in C[0,1]$. The kernels $k_{1}(x, s), \quad k_{2}(x, s)$ are of the form:

$$
|s-x|^{-\alpha} 0<\alpha<1 \text { and } \log |s-x|
$$

if $\lambda \in \mathbb{R}$ and $\lambda \neq 0$, there exists a unique function $u \in C[0,1]$ solution of (1).

\section{On the q.i. Splines}

In the following we recall the necessary background on q.i. splines space. 
Let $X_{m}:=\left\{x_{0, m}=a<x_{1, m}<\cdots<x_{m, m}<x_{m+1, m}=b\right\}$ be a partition of the interval $J:=[a, b]$ with

$H_{m}:=\max _{0 \leq j \leq m}\left(x_{j+1, m}-x_{j, m}\right), H_{m} \rightarrow 0$ as $m \rightarrow \infty$ and let $\left\{d_{j}: j=0, \cdots, m+1\right\}$ be a vector of positive integers where $d_{0}=d_{m+1}=p \quad(p \geq 2)$ and $d_{j}<p, \quad j=1, \cdots, m$.

We set $n+p:=\sum_{j=0}^{m+1} d_{j}$ and define

$\Pi_{n}=\left\{t_{i}: i=1, \cdots, n+p\right\}$ as the nondecreasing sequence obtained from $X_{m}$ by repeating $x_{j, m}$ exactly $d_{j}$ times, $j=0, \cdots, m+1$.

$\Pi_{n}$ is the set of knots defining the $p$-order polynomial spline space $S_{p, \Pi_{n}}$. Any spline space $S_{p, \Pi_{n}}$ based on the set $X_{m}$ is said to be locally uniform if:

$$
\frac{x_{j+1, m}-x_{j, m}}{x_{k+1, m}-x_{k, m}} \leq A, \quad k=j \pm 1, j=1, \cdots, m-1
$$

where $A \geq 1$ does not depend on $j$ nor $m$.

Let consider as a basis for the spline space $S_{p, \Pi_{n}}$ the set of the normalized B-splines $B_{i, p}(i=1, \cdots, n)$ of order $p$ defined by the following recurrence relation:

$$
\begin{gathered}
B_{i, p}(x)=\frac{x-t_{i}}{t_{i+p-1}-t_{i}} B_{i, p-1}(x)+\frac{t_{i+p}-x}{t_{i+p}-t_{i+1}} B_{i+1, p-1}(x) \\
B_{i, 1}(x)= \begin{cases}1, & t_{i} \leq x<t_{i+1} \\
0, & \text { otherwise }\end{cases}
\end{gathered}
$$

To the aim to define q.i. spline operators we consider a set $T$ of nodes $\tau_{i j} \quad(i=1, \cdots, n ; j=1, \cdots, p)$ belonging for each $i=1, \cdots, n$ to a subset of $\left[t_{i}, t_{i+p}\right]$ and such that $\tau_{i j} \neq \tau_{i h}$ for $j \neq h$.

In [7] and in [13] the following sets are suggested:

$$
\left.\begin{array}{c}
T_{1}: \tau_{i j}:=t_{i}+(j-1) \frac{t_{i+p}-t_{i}}{p-1}, \quad j=1, \cdots, p, i=1, \cdots, n \\
T_{2}: \tau_{i j}:=t_{i}+\left(j-\frac{1}{2}\right) \frac{t_{i+p}-t_{i}}{p}, \quad j=1, \cdots, p, i=1, \cdots, n \\
T_{3}: \tau_{i j}:=t_{i+j-1}, \quad j=1, \cdots, p, i=p+1, \cdots, n-p \\
T_{4}: \tau_{i j}:=t_{i+j}, \quad j=1, \cdots, p, i=p+1, \cdots, n-p \\
T_{5}: \tau_{i j}:=\frac{t_{i+p-1}+t_{i+j}}{2}, \quad j=1, \cdots, p, i=p+1, \cdots, n-p \\
T_{6}:\left\{\begin{array}{l}
\tau_{i 1}:=\zeta_{i}, i=1, \cdots, n \\
\tau_{i 2}:=\zeta_{i-1}, \tau_{i 3}:=\zeta_{i+1}, \cdots, \\
\tau_{i p}:=\zeta_{i-(-1)^{p}}\left[\frac{p}{2}\right]
\end{array}\right]=\left[\frac{p}{2}\right]+1, \cdots, n-\left[\frac{p}{2}\right]
\end{array}\right\}
$$

where $\zeta_{i}=\frac{t_{i+1}+\cdots+t_{i+p-1}}{p-1}, \quad i=1, \cdots, n$, with a suitable choice of the nodes for the remaining values of $i$ : in
$T_{3}-T_{5}$ as in [7] and in $T_{6}$ as in [13].

Let now consider the operator $\mathcal{S}_{n}: C[a, b] \rightarrow S_{p, \Pi_{n}}$ so defined:

$$
\mathcal{S}_{n} g(x):=\sum_{i=1}^{n} B_{i, p}(x) \sum_{j=1}^{p} v_{i j} g\left(\tau_{i j}\right),
$$

where

$$
\begin{gathered}
v_{i j}:=\sum_{\mu=j}^{p} \frac{\alpha_{i \mu}}{\prod_{\substack{s=1 \\
s \neq j}}^{\mu}\left(\tau_{i j}-\tau_{i s}\right)} \\
\alpha_{i j}:=\sum_{k=1}^{j}(-1)^{j-k} \frac{(k-1) !(p-k) !}{(p-1) !} c_{i, k-1} d_{i, j-k},
\end{gathered}
$$

with $c_{i, k-1}=\operatorname{symm}_{k-1}\left(t_{i+1}, \cdots, t_{i+p-1}\right)$, $d_{i, j-k}=\operatorname{symm}_{j-k}\left(\tau_{i 1}, \cdots, \tau_{i, j-1}\right)$ (see [6]).

In the following we use in (4) $p=4, d_{j}=1$

$(j=1, \cdots, m), \quad d_{0}=d_{m+1}=4$, and $\tau_{i j} \in T_{7}$, where $T_{7}$ is defined as $T_{6}$ in (3) with the remaining nodes suitably chosen as: $\tau_{1,4}:=\frac{\tau_{1,2}+\tau_{1,3}}{2}, \tau_{2,4}:=\zeta_{1}$, $\tau_{n, 4}:=\frac{\tau_{n, 2}+\tau_{n, 3}}{2}, \tau_{n-1,4}:=\zeta_{n}$. Consequently we obtain that the following properties for the operator $\mathcal{S}_{n}$ hold:

$-\mathcal{S}_{n}$ reproduces exactly a polynomial of $p-1$ degree that is $[6]$ :

$$
\mathcal{S}_{n} P=P, P \in \mathcal{P}_{p}
$$

-as $\tau_{i j}$ chosen in $T_{7}$ belong to a proper subinterval of $\left[t_{i}, t_{i+p}\right]$, for all $i$ and $j=1, \cdots, p$, then $\mathcal{S}_{n}$ is a projection operator [14], that is:

$$
\mathcal{S}_{n} S=S, S \in S_{p, \Pi_{n}} .
$$

\section{The Numerical Model}

The Equation (1) can be reduced to the following compact form

$$
[\lambda I+\tilde{\mathcal{K}}] u=f,
$$

where:

$-I$ is the identity operator;

$-\tilde{\mathcal{K}}$ is the following operator:

$$
\tilde{\mathcal{K}} g=\tilde{\mathcal{K}}_{1} g+\mathcal{K}_{2} g
$$

where:

$$
\begin{gathered}
\tilde{\mathcal{K}}_{1} g(x):=-\int_{0}^{1} \tilde{k}_{1}(x, s) g(s) \mathrm{d} s, \quad x \in[0,1] \\
\mathcal{K}_{2} g(x):=-\int_{0}^{1} k_{2}(x, s) g(s) \mathrm{d} s, \quad x \in[0,1] \\
\tilde{k}_{1}(x, s):= \begin{cases}k_{1}(x, s) & \text { if } 0 \leq s \leq x \\
0 & \text { if } s>x\end{cases}
\end{gathered}
$$


Let

$$
r_{n}=[\lambda I+\tilde{\mathcal{K}}] \mathcal{S}_{n} u-f
$$

where $\mathcal{S}_{n} u$ is in (4). If we collocate (9) in a set of $n p$ points, we could completely define $\mathcal{S}_{n} u$. Neverthless the choice of the set $T_{7}$ of the nodes and the definition of $v_{i j}$ as in (5) allow, by the algorithm, to reduce the dimension of the collocation system. Consequently the collocation system on a set of distinct collocation points $\xi_{k}(k=1,2, \cdots, n)$, chosen in $(0,1)$, is the following one

$$
r_{n}\left(\xi_{k}\right)=[\lambda I+\tilde{\mathcal{K}}] \mathcal{S}_{n} u\left(\xi_{k}\right)-f\left(\xi_{k}\right)=0, \quad k=1,2, \cdots, n
$$

We assume as an approximation of the solution of (1) the following function belonging to $S_{p, \Pi_{n}}$ spline space

$$
w_{n}(x)=\sum_{i=1}^{n} B_{i, p}(x) \sum_{j=1}^{p} v_{i j} \bar{u}_{i},
$$

where the $\bar{u}_{i}$ are the approximated values of function $u$ in $\tau_{i 1}$, obtained from the collocation system (10).

Finally we observe that to complete the algorithm we must to compute the coefficients of the collocation system and then to evaluate the following integrals:

$$
\begin{aligned}
& \mathcal{J}_{1} \mathcal{S}_{n} u=\int_{0}^{\xi_{k}} k_{1}\left(\xi_{k}, s\right) \sum_{i=1}^{n} \sum_{j=1}^{p} v_{i j} u\left(\tau_{i j}\right) B_{i, p}(s) \mathrm{d} s \\
& \mathcal{J}_{2} \mathcal{S}_{n} u=\int_{0}^{1} k_{2}\left(\xi_{k}, s\right) \sum_{i=1}^{n} \sum_{j=1}^{p} v_{i j} u\left(\tau_{i j}\right) B_{i, p}(s) \mathrm{d} s
\end{aligned}
$$

which lead to the determination of

$$
\mathcal{I}_{1} B_{i, 1}=\int_{0}^{\xi_{k}} k_{1}\left(\xi_{k}, s\right) B_{i, 1}(s) s^{p-1} \mathrm{~d} s
$$

and

$$
\mathcal{I}_{2} B_{i, 1}=\int_{0}^{1} k_{2}\left(\xi_{k}, s\right) B_{i, 1}(s) s^{p-1} s \mathrm{~d} s
$$

with $i=1, \cdots, n$.

The computation of (13) and (14) is carried out through a closed analytical form, when possible.

Otherwise we substitute (11) and (12) with:

$$
\mathcal{L}_{1} \mathcal{S}_{n} k_{1} u=\int_{0}^{\xi_{k}} \sum_{i=1}^{n} \sum_{j=1}^{p} k_{1}\left(\xi_{k}, \tau_{i j}\right) v_{i j} u\left(\tau_{i j}\right) B_{i, p}(s) \mathrm{d} s
$$

and

$$
\mathcal{L}_{2} \mathcal{S}_{n} k_{2} u=\int_{0}^{1} \sum_{i=1}^{n} \sum_{j=1}^{p} k_{2}\left(\xi_{k}, \tau_{i j}\right) v_{i j} u\left(\tau_{i j}\right) B_{i, p}(s) \mathrm{d} s
$$

respectively.

\section{On the Convergence}

In this Section we study the convergence of $w_{n} \rightarrow u$ for $n \rightarrow \infty$.
Let $E=\left\{C[0,1],\|\circ\|_{\infty}\right\}$ be a Banach space on $\mathbb{R}$ with

$$
\|y\|=\|y\|_{\infty}:=\max \{|y(t)| ; 0 \leq t \leq 1 \forall y \in C[0,1]\}
$$

and the norm of the operator $\mathcal{T}: E \rightarrow E$

$$
\|\mathcal{T}\|=\sup _{\|y\|_{\infty} \leq 1}|\mathcal{T} y|
$$

Lemma 1: Let $\left\{\Pi_{n}\right\}$ be a sequence of l.u. partitions. The operator $\mathcal{S}_{n}: C[0,1] \rightarrow S_{p, \Pi_{n}}$ is a bounded compact operator and such that for $g \in C[0,1]$

$$
\left\|\mathcal{S}_{n} g-g\right\|_{\infty} \rightarrow 0 \text { as } n \rightarrow \infty .
$$

\section{Proof:}

As $\alpha_{i j}$ in (6) for all $i, j$ are bounded and $\prod_{\substack{s=1 \\ s \neq j}}^{\mu}\left(\tau_{i j}-\tau_{i s}\right)$ in (5) has a minimum (see [7]), then $\mathcal{S}_{n}$ defined in (4) is a bounded operator.

Moreover, as $\tau_{i j} \in T_{7}(j=1, \cdots, p$, for all $i)$, the thesis follows (see Theorem A in [7]).

Furthermore it can be noted that the kernel

$$
\tilde{k}(x, s)=\tilde{k}_{1}(x, s)+k_{2}(x, s)
$$

satisfies the following properties:

1) $\tilde{k}(x, s)$ is Riemann-integrable as function of $s$, for all $x \in[0,1]$.

2) $\lim _{x \rightarrow x^{\prime}} \int_{0}^{1}\left|\tilde{k}\left(x^{\prime}, s\right)-\tilde{k}(x, s)\right| \mathrm{d} s=0$, for $x^{\prime}, x \in[0,1]$.

3) $\max _{x \in[0,1]} \int_{0}^{1}|\tilde{k}(x, s)| \mathrm{d} s<\infty$.

Consequently the operator $\tilde{\mathcal{K}} u$ in (8) is a bounded compact operator.

Moreover this condition states the existence and uniqueness of the solution of (1) (see [15]), that is the existence of $(\lambda I+\tilde{\mathcal{K}})^{-1}$.

Lemma 2: Let $\left\{\Pi_{n}\right\}$ be a sequence of l.u. partitions. Let consider the sequence of bounded and projection operators $\mathcal{S}_{n}$ in (7): $C[0,1] \rightarrow S_{p, \Pi_{n}}$, it follows that:

$$
\left\|\tilde{\mathcal{K}}-\mathcal{S}_{n} \tilde{\mathcal{K}}\right\| \rightarrow 0 \text { as } n \rightarrow \infty .
$$

Proof: As $\tilde{\mathcal{K}}: C[0,1] \rightarrow C[0,1]$ is a compact operator and since (15) holds, then (16) is proved. $\square$

Theorem 1: Let $\left\{\Pi_{n}\right\}$ be a sequence of l.u. partitions. Let consider the bounded and projection operator $\mathcal{S}_{n}: C[0,1] \rightarrow S_{p, \Pi_{n}}$. For all $n$ sufficiently large $(n \geq N)$ the operator $\left(\lambda I+\mathcal{S}_{n} \tilde{\mathcal{K}}\right)^{-1}: C[0,1] \rightarrow C[0,1]$ exists. Moreover it is uniformly bounded, that is:

$$
\sup _{n \geq N}\left\|\left(\lambda I+\mathcal{S}_{n} \tilde{\mathcal{K}}\right)^{-1}\right\| \leq M<\infty
$$

and

$$
\left\|u-w_{n}\right\| \leq \lambda\left\|\left(\lambda I+\mathcal{S}_{n} \tilde{\mathcal{K}}\right)^{-1}\right\|\left\|u-\mathcal{S}_{n} u\right\|
$$


that is $w_{n} \rightarrow u$ for $n \rightarrow \infty$.

Proof: from (10) (see [15])

$$
\mathcal{S}_{n} r_{n}(x)=0
$$

As $\mathcal{S}_{n}$ is bounded projection operator (19) becomes:

$$
\mathcal{S}_{n}[\lambda I+\tilde{\mathcal{K}}] w_{n}-\mathcal{S}_{n}[\lambda I+\tilde{\mathcal{K}}] u=0
$$

From (20) we can easily obtain

$$
u-w_{n}=\lambda\left(\lambda I+\mathcal{S}_{n} \tilde{\mathcal{K}}\right)^{-1}\left(u-\mathcal{S}_{n} u\right)
$$

Now we must prove the existence and boundedness of $\left(\lambda I+\mathcal{S}_{n} \tilde{\mathcal{K}}\right)^{-1}$.

By simple algebraic steps it follows that

$$
\lambda I+\mathcal{S}_{n} \tilde{\mathcal{K}}=(\lambda I+\tilde{\mathcal{K}})\left[I+(\lambda I+\tilde{\mathcal{K}})^{-1}\left(\mathcal{S}_{n} \tilde{\mathcal{K}}-\tilde{\mathcal{K}}\right)\right]
$$

It is necessary to ensure that $I+(\lambda I+\tilde{\mathcal{K}})^{-1}\left(\mathcal{S}_{n} \tilde{\mathcal{K}}-\tilde{\mathcal{K}}\right)$ has an inverse bounded operator.

As, from Lemma 2, $\left\|\tilde{\mathcal{K}}-\mathcal{S}_{n} \tilde{\mathcal{K}}\right\| \rightarrow 0$ as $n \rightarrow \infty$, we can find an integer $N$ such that

$$
\varepsilon_{N}=\sup _{n \geq N}\left\|\tilde{\mathcal{K}}-\mathcal{S}_{n} \tilde{\mathcal{K}}\right\|<\frac{1}{\left\|(\lambda I+\tilde{\mathcal{K}})^{-1}\right\|}
$$

Consequently, adapting Theorem 3.1.1. in [15], we obtain for $n \geq N$

$$
\left\|\left[I+(\lambda I+\tilde{\mathcal{K}})^{-1}\left(\mathcal{S}_{n} \tilde{\mathcal{K}}-\tilde{\mathcal{K}}\right)\right]^{-1}\right\| \leq \frac{1}{1-\varepsilon_{N}\left\|(\lambda I+\tilde{\mathcal{K}})^{-1}\right\|}
$$

and we can conclude that $\left[I+(\lambda I+\tilde{\mathcal{K}})^{-1}\left(\mathcal{S}_{n} \tilde{\mathcal{K}}-\tilde{\mathcal{K}}\right)\right]^{-1}$ exists and it is bounded.

Considering that from (22)

$$
\begin{aligned}
& \left\|\left(\lambda I+\mathcal{S}_{n} \tilde{\mathcal{K}}\right)^{-1}\right\| \\
\leq & \left\|(\lambda I+\tilde{\mathcal{K}})^{-1}\right\|\left\|\left[I+(\lambda I+\tilde{\mathcal{K}})^{-1}\left(\mathcal{S}_{n} \tilde{\mathcal{K}}-\tilde{\mathcal{K}}\right)\right]^{-1}\right\|
\end{aligned}
$$

and consequently

$$
\left\|\left(\lambda I+\mathcal{S}_{n} \tilde{\mathcal{K}}\right)^{-1}\right\| \leq \frac{\left\|(\lambda I+\tilde{\mathcal{K}})^{-1}\right\|}{1-\varepsilon_{N}\left\|(\lambda I+\tilde{\mathcal{K}})^{-1}\right\|}=M<\infty
$$

then (17) is proved.

From (21) it follows (18), that is $\left\|u-w_{n}\right\| \rightarrow 0$, $n \rightarrow \infty$, exactly with the same rate of convergence as $\left\|u-\mathcal{S}_{n} u\right\|$ does (see Lemma 1).

Remark 1. The assumption of the $\tau_{i j}$ points in $T_{7}$ ( $j=1, \cdots, p$ for all $i$ ) is decisive for the convergence. Moreover we underline that the choice of the $\tau_{i j} \in T_{7}$ arises from a compromise between two practical different constraints: maximizing the polynomial precision of the approximation and minimizing the collocation system order (see [13]).

\section{Numerical Results}

In what follows we present some numerical results for some Volterra-Fredholm integral Equations (1), by using the numerical method presented above. The algorithm is implemented by MATLAB 7.3.

We consider the following equation:

$$
\begin{aligned}
& \lambda u(x)=f(x)+\int_{0}^{x} k_{1}(x, s) u(s) \mathrm{d} s+\int_{0}^{1} k_{2}(x, s) u(s) \mathrm{d} s, \\
& x \in[0,1] .
\end{aligned}
$$

In Tables 1 and $\mathbf{2}$ we show the results obtained with the choice $k_{1}(x, s)=k_{2}(x, s)=-|s-x|^{-1 / 2}$ and $k_{1}(x, s)=\log |s-x|, \quad k_{2}(x, s)=1$, respectively, and $\lambda=$ 1. In particular, the polynomial exactness of the method till third degree is tested.

In the interval $[0,1]$ we choose $m=11$ points $x_{j}$ $(j=1, \cdots, 9)$, all simple.

The unknown function is approximated in 13 nodes belonging to $[0,1]$. For brevity in Table 1 we indicate the mean of the absolute values of the errors evaluated in the interval.

In Table 3 we show the results obtained with the choice $k_{1}(x, s)=-|s-x|^{-1 / 2}, \quad k_{2}(x, s)=0, \quad u(x)=\sqrt{x}$, $f(x)=\sqrt{x}+\frac{\pi}{2} x$ with different number of nodes in

Table 1. $k_{1}(x, s)=k_{2}(x, s)=-|s-x|^{-1 / 2}$.

\begin{tabular}{ccc}
\hline$f(x)$ & $u(x)$ & $E R R$ \\
\hline$x+2 / 3\left[\sqrt{1-x}(1+2 x)+4 x^{3 / 2}\right]$ & $x$ & $4.45 \times 10^{-16}$ \\
$x^{3}+2 / 7\left[\sqrt{1-x}\left(1+6 x / 5+8 x^{2}(1+2 x) / 5\right)+32 x^{7 / 2} / 5\right]$ & $x^{3}$ & $9.08 \times 10^{-17}$ \\
$x^{4}+2 / 9\left[\sqrt{1-x}\left(1+8 x / 7+48 x^{2} / 35+64 x^{3}(1+2 x) / 35\right)+256 x^{7 / 2} / 35\right]$ & $x^{4}$ & $1.88 \times 10^{-5}$ \\
\hline
\end{tabular}


Table 2. $k_{1}(x, s)=\log |s-x|, \quad k_{2}(x, s)=1$.

\begin{tabular}{ccc}
\hline$f(x)$ & $u(x)$ & ERR \\
\hline $2+x \log x-x$ & 1 & $4.44 \times 10^{-15}$ \\
$x^{2}+x^{3}(\log x-11 / 6) / 3+1 / 3$ & $x^{2}$ & $1.02 \times 10^{-15}$ \\
$x^{4}+x^{5}(\log x-137 / 60) / 5+1 / 5$ & $x^{4}$ & $7.41 \times 10^{-5}$ \\
\hline
\end{tabular}

Table 3. $k_{1}(x, s)=-|s-x|^{-1 / 2}, \quad k_{2}(x, s)=0$.

\begin{tabular}{ccccc}
\hline$x$ & $m=11$ & $m=21$ & $m=41$ & $m=101$ \\
\hline 0.1 & $7.6 \times 10^{-3}$ & $1.1 \times 10^{-3}$ & $7.1 \times 10^{-5}$ & $6.9 \times 10^{-6}$ \\
0.5 & $6.6 \times 10^{-5}$ & $1.1 \times 10^{-5}$ & $3.4 \times 10^{-6}$ & $8.4 \times 10^{-7}$ \\
1 & $1.8 \times 10^{-5}$ & $3.0 \times 10^{-6}$ & $1.2 \times 10^{-6}$ & $3.2 \times 10^{-7}$ \\
\hline
\end{tabular}

$[0,1]$. The results denote that the use of the cubic q.i. splines with a suitable densification of the nodes near 0 , using the graded mesh in [16], leads to absolute errors of the same order as the results obtained in [16] with the choice of quadratic nodal spline and the same meshes.

\section{REFERENCES}

[1] T. N. E. Greville, "Spline Functions, Interpolation and Numerical Quadrature," In: Mathematical Methods for Digital Computers, Wiley, New York, 1967, pp. 156-168.

[2] C. Dagnino, "Product Integration of Singular Integrals Based on Cubic Spline Interpolation at Equally Spaced Nodes," Numerical Mathematics, Vol. 57, No. 1, 1990, pp. 97-104. http://dx.doi.org/10.1007/BF01386400

[3] A. P. Orsi, "Spline Approximation for Cauchy Principal Value Integrals," JCAM, Vol. 30, No. 1, 1990, pp. 191201.

[4] P. Rabinowitz, "Numerical Integration Based on Approximating Splines," Journal of Computational and Applied Mathematics, Vol. 33, No. 1, 1990, pp. 73-83. http://dx.doi.org/10.1016/0377-0427(90)90257-Z

[5] I. J. Schoenberg, "On Spline Functions," Inequalities (Symposium at Write-Patterson Air Force Base), Academic Press, New York, 1967, pp. 255-291.

[6] T. Lyche and L. L. Schumaker, "Local Spline Approximation Methods," Journal of Approximation Theory, Vol. 15, No. 4, 1975, pp. 294-325. http://dx.doi.org/10.1016/0021-9045(75)90091-X
[7] C. Dagnino and P. Rabinowitz, "Product Integration of Singular Integrands Using Quasi-Interpolatory Splines," Computers \& Mathematics with Applications, Vol. 33, No. 1, 1997, pp. 59-67. http://dx.doi.org/10.1016/S0898-1221(96)00219-2

[8] C. Dagnino and E. Santi, "Quadrature Based on QuasiInterpolating Spline-Projectors for Product Singular Integration," Babes-Bolyai, Mathematica, Vol. XLI, 1996, pp. 35-47.

[9] F. Caliò, E. Marchetti and P. Rabinowitz, "On the Numerical Solution of the Generalized Prandtl Equation Using Variation-Diminishing Splines," Journal of Computational and Applied Mathematics, Vol. 60, No. 3, 1995, pp. 297-307.

http://dx.doi.org/10.1016/0377-0427(94)00024-U

[10] F. Caliò and E. Marchetti, "An Algorithm Based on q.i. Modified Splines for Singular Integral Models," Computers \& Mathematics with Applications, Vol. 41, No. 12, 2001, pp. 1579-1588. http://dx.doi.org/10.1016/S0898-1221(01)00123-7

[11] F. Caliò, M. V. Fernandéz Muñoz and E. Marchetti, "Direct and Iterative Methods for the Numerical Solution of Mixed Integral Equations," Applied Mathematics and Computation, Vol. 216, No. 12, 2010, pp. 3739-3746. http://dx.doi.org/10.1016/j.amc.2010.05.032

[12] F. Caliò, A. I. Garralda-Guillem, E. Marchetti and M. R. Galán, "About Some Numerical Approaches for Mixed Integral Equations," Applied Mathematics and Computation, Vol. 219, No. 2, 2012, pp. 464-474. http://dx.doi.org/10.1016/j.amc.2012.06.013

[13] V. Demichelis, "Quasi-Interpolatory Splines Based on Schoenberg Points," Mathematics of Computation, Vol. 65, No. 215, 1996, pp. 1235-1247. http://dx.doi.org/10.1090/S0025-5718-96-00728-4

[14] C. de Boor, "Spline Approximation by Quasi Interpolants," Journal of Approximation Theory, Vol. 8, No. 1, 1973, pp. 19-45. http://dx.doi.org/10.1016/0021-9045(73)90029-4

[15] K. E. Atkinson, "The Numerical Solution of Integral Equations of the Second Kind," Cambridge University Press, Cambridge, 1997.

[16] C. Dagnino, V. Demichelis and E. Santi, “A Nodal Spline Collocation Method for Weakly Singular Volterra Integral Equations," Babes-Bolyai, Mathematica, Vol. XLVIII, 2003, pp. 71-81. 